\title{
A Service Evaluation to Determine the Efficacy of a Specialist Service for Adults with Attention-Deficit Hyperactivity Disorder in Norfolk and Waveney
}

\author{
Danielle Wilson ${ }^{1}$ and Lisa Riches ${ }^{1}$ \\ ${ }^{1}$ Norfolk and Suffolk NHS Foundation Trust
}

November 26, 2020

\begin{abstract}
Rationale, aims \& objectives: This evaluation was carried out in order to determine the efficacy of the Norfolk and Waveney Adult ADHD Service. With the service not commissioned to offer non-pharmacological support, and with a gap in the research literature, evidence on whether the service model was effective at reducing the impact of ADHD symptoms on service users was needed in order to justify longer term commissioning. Method: Case notes of 113 individuals going through their medication titration process were observed. Scores from the Weiss Functional Impairment Rating Scale (WFIRS), which was routinely collected in the service pre- and post- titration onto ADHD medication, were recorded for comparison. Results: A T test revealed significant improvements in functioning across all domains of the WFIRS after successful titration onto ADHD medication. However, $19.5 \%$ of the sample disengaged from the service prior to completing titration. A further $12.5 \%$ of the sample did not complete titration due to various factors discussed. Conclusion: While medication has been shown to be effective at improving impairment of functioning in adults with ADHD, high levels of disengagement suggest that more needs to be offered to this population.
\end{abstract}

A Service Evaluation to Determine the Efficacy of a Specialist Service for Adults with Attention-Deficit Hyperactivity Disorder in Norfolk and Waveney

An Evaluation of an Adult ADHD Service

Authors: Danielle Wilson ${ }^{1}$, Lisa Riches ${ }^{2}$

Rationale, aims \& objectives: This evaluation was carried out in order to determine the efficacy of the Norfolk and Waveney Adult ADHD Service. With the service not commissioned to offer non-pharmacological support, and with a gap in the research literature, evidence on whether the service model was effective at reducing the impact of ADHD symptoms on service users was needed in order to justify longer term commissioning.

Method: Case notes of 113 individuals going through their medication titration process were observed. Scores from the Weiss Functional Impairment Rating Scale (WFIRS), which was routinely collected in the service pre- and post- titration onto ADHD medication, were recorded for comparison.

Results: A T test revealed significant improvements in functioning across all domains of the WFIRS after successful titration onto ADHD medication. However, $19.5 \%$ of the sample disengaged from the service prior to completing titration. A further $12.5 \%$ of the sample did not complete titration due to various factors discussed.

Conclusion: While medication has been shown to be effective at improving impairment of functioning in adults with ADHD, high levels of disengagement suggest that more needs to be offered to this population. 
Keywords - Attention-Deficit Hyperactivity Disorder; Adult; Service; Evaluation

\section{Introduction}

\subsection{The Norfolk and Waveney Adult ADHD Service}

The Norfolk and Waveney Adult ADHD Service is a primary care interface service, commissioned under the Norfolk and Suffolk NHS Foundation Trust. They offer assessment, diagnosis and titration onto a suitable ADHD medication. Once stabilised with drug therapy, service users are transferred back to the care of their general practitioner (GP), under a shared care agreement. Those on ADHD medication are then invited back for an annual medication review. Those not on medication are discharged from the service.

\subsection{Background}

Attention-Deficit/Hyperactivity Disorder (ADHD), historically thought of as a problem seen in childhood, is now recognised as an adult neurodevelopmental disorder ${ }^{1}$. There is evidence that impairments relating to ADHD persist into adulthood, with two thirds of children diagnosed with ADHD continuing to either have full or partial traits beyond the age of $18^{2}$.

Numerous longitudinal and cross-sectional studies have illustrated that, when compared to control groups, ADHD is associated with lower academic attainment ${ }^{3}$ and occupational performance ${ }^{4}$, as well as increased likelihood of health problems ${ }^{5}$, psychiatric comorbidity ${ }^{6}$, marital/relationship issues ${ }^{7}$, and imprisonment ${ }^{8}$.

Follow-up studies suggest treatment with medication significantly improves an individuals' quality of life ${ }^{9}$. However, in previous controlled trials of pharmacotherapy, $20 \%$ to $50 \%$ of adults were considered 'nonresponders', due to insufficient symptom reduction or an inability to tolerate medication ${ }^{10}$. This raises questions as to whether a service model that only offers pharmacological intervention is effective at reducing the impact of ADHD symptoms on service users' functioning.

The 2018 NICE guidelines ${ }^{11}$ alongside other published research ${ }^{12,13}$ provide an evidence base for the need for services for adults with ADHD, as well as clear recommendations for the assessment and management for ADHD in adulthood. NICE ${ }^{11}$ recommends medication to adults with ADHD if their ADHD symptoms are still causing a significant impairment in at least 1 domain after environmental modifications have been implemented and reviewed. Non-pharmacological treatment should be considered if the service user has made an informed choice not to have medication, has difficulty adhering to medication, or has found medication to be ineffective or they cannot tolerate it. A combination of non-pharmacological treatment and medication should be considered for service users who have benefitted from medication but whose symptoms are still causing a significant impairment in at least 1 domain.

Recent evaluations of Adult ADHD Services in the UK ${ }^{14,15}$ have focused on adherence to NICE guidelines, but to the best of our knowledge, none have focused on service user outcomes.

\section{Method}

This project was classified and approved as a service evaluation by the Norfolk and Suffolk NHS Foundation Trust (NSFT) Research and Development Department. We utilised an outcome measure which was routinely used in the service, the Weiss Functional Impairment Rating Scale (WFIRS) ${ }^{16}$. Scores before and after titration onto ADHD medication were recorded and compared. As explained by Ramsay ${ }^{17}$, it can be useful to measure functional impairment in order to illustrate the effects of ADHD on one's life and to justify the need for treatment, to track treatment progress and optimise treatments for adults with ADHD. The present study aimed to evaluate the efficacy of a specialist adult ADHD service, by assessing the difference (if any) in service users' self-reported functional impairment before and after titration onto ADHD medication.

The WFIRS ${ }^{16}$ is a self-report scale, designed to assess ADHD-related functional impairment in seven domains that are clinically relevant to ADHD in adulthood, including home, self-concept, learning \& work, activities of daily living (ADLs), social activities, and risky activities ${ }^{16,18,19}$. These domains are likely to represent the target for treatment, meaning the use of the scale before and after treatment can determine improvements 
in the service user's ADHD, as well as whether functional difficulties have been alleviated ${ }^{19}$. In addition to this, the WFIRS demonstrates robust internal reliability, cross-informant agreement on par or superior to other measures of ADHD symptomatology and impairment, and concurrent validity ${ }^{19}$.

We monitored the case notes of service users who were seen by a Non-Medical Prescriber between $1^{\text {st }}$ January 2019 and $31^{\text {st }}$ March 2019, regardless to where they were in the titration process. Service users whom were already on ADHD medication at the time of their initial appointment (and therefore classed as annual medication reviews) were excluded from the sample. A total of 159 service users were seen by prescribers between $1^{\text {st }}$ January 2019 and $31^{\text {st }}$ March 2019, with 46 of these being excluded as they were annual medication reviews. This left a sample of 113 service users for the evaluation.

Demographic information (age and gender) was collected, as well as their engagement status at the time of final data collection (30 ${ }^{\text {th }}$ August 2019). Mean scores were calculated for each domain of the WFIRS (18 Weiss 2000), pre- and post-titration to allow for comparison between domains and overall.

\section{Results}

\subsection{Demographics}

Of the 113 people included in the evaluation, 74 were males and 39 were females, with an age range of 18-56 (and a mean of 31.02 and standard deviation of 10.28). 43 service users (38\% of the sample) were previously undiagnosed and had been given a diagnosis of ADHD prior to their titration. The remaining 70 service users $(62 \%)$ had received a diagnosis prior to the present episode of care.

\subsection{Engagement}

At the end of data collection, 46 service users ( $40.7 \%$ of the sample) had been successfully titrated onto medication and discharged back to the care of their GP. A further 31 service users (27.4\% of the sample) were still being titrated.

The remaining 36 service users (31.9\% of the sample) did not complete titration and had been discharged from the service. Reasons for this were either the service user disengaging from the service (i.e. not attending appointments to complete titration) (19.5\% of the sample), or not remaining on ADHD medication due to intolerable side effects $(2.7 \%)$, a deterioration in their mental health which warranted a referral to another team $(2.7 \%)$, substance abuse $(0.9 \%)$ or concerns over cardiac health $(3.5 \%)$. A further 3 service users $(2.7 \%)$ decided against trialling ADHD medication before titration began. This cohort therefore did not complete post-treatment measures. Any pre-treatment measures recorded were included in the analyses.

As illustrated in table 1, when comparing the engagement of those who had recently been given an ADHD diagnosis and those previously diagnosed, a much higher proportion of those recently diagnosed had disengaged from the service (25.6\% compared to $15.7 \%)$. However, the small sample size makes it difficult to draw any conclusions from this.

\section{Weiss Functional Impairment Rating Scale (WFIRS)}

Of the 113 service users in the sample, 69 had completed a pre-medication WFIRS. This gives a completion rate of $61.1 \%$. By the cut-off date, 46 people had completed titration, with 26 of these completing the post-titration WFIRS. This gives a completion rate of $56.5 \%$ (or $23 \%$ of the overall sample).

Mean scores for each domain were calculated, with questions marked 'not applicable' being excluded. This scoring method enables a comparison between each domain, both pre- and post-titration. Table 2 illustrates the mean scores and standard deviations of each domain in the WFIRS, both pre- and post-titration on ADHD medication. The mean overall score pre-titration was $1.66(\mathrm{SD}=0.48)$, which decreased to 0.82 $(\mathrm{SD}=0.51)$ post-titration.

The results indicate that ADHD medication does significantly reduce the level of impairment experienced in all domains of the WFIRS. All areas were robust to Bonferroni correction for multiple comparisons where $\alpha$ was set to $0.5 / 7$ or 0.007 . Additionally, self-concept had the highest rated impairment $(\mathrm{M}=2.46, \mathrm{SD}=0.62)$, 
and risky activities had the lowest $(0.95, \mathrm{SD}=0.79)$ before titration. Despite significant differences with post-titration mean scores, the highest and lowest rated domains for impairment remained the same.

\subsection{Did people that disengaged have higher functioning compared those that completed titra- tion?}

22 service users disengaged from the service prior to completing their titration. This means they did not attend an appointment or contact the service to rearrange an appointment. At the time of the evaluation, those that did not attend an appointment without prior notice were contacted by phone and sent an opt in letter requesting them to contact the service to rearrange their appointment. If the service user did not make contact to rearrange their appointment, or they missed a second appointment, they were discharged due to disengagement.

Of the 22 discharged due to disengagement, 18 had pre-titration WFIRS. 46 service users completed titration, with 26 having pre-titration WFIRS. As illustrated in table 3, results indicate that when applying a Bonferroni correction for multiple comparisons where $\alpha$ is set to $0.5 / 7$ or 0.007 , those that disengaged from the service did not report significantly different levels of impairment across the 6 domains of the WFIRS compared to those that completed titration.

\section{Discussion}

To the best of our knowledge, this is the first evaluation of the effectiveness of a specialist adult ADHD service in relation to improvements of functional impairment, pre- and post-titration onto ADHD medication. The results indicate that overall, ADHD medication is effective at reducing the functional impairment of adults with ADHD in all domains of the WFIRS, which is consistent with similar research in this area ${ }^{20}$. This evidence has contributed to longer term commissioning of the service. However, the large proportion of service users either disengaging from treatment (19.5\% of the sample), being assessed as not suitable for medication (12.4\% of the sample) or choosing not to have medication ( $2.7 \%$ of the sample), highlights a significant service gap and the need to offer an alternative intervention for the management of ADHD. This endorses NICE guidelines ${ }^{11}$, which recommends non-pharmacological treatment for adults with ADHD who have either made an informed choice not to have medication, have difficulty adhering to medication; or found medication to be ineffective/intolerable.

The present study also found that the level of functional impairment reported by people that disengaged from the service did not significantly differ to those that completed titration, suggesting that this was not a factor in people disengaging from treatment. It is possible that those who disengaged could have done so due to unmanaged ADHD symptoms (e.g. the organisational skills needed to attend regular appointments over several months). This further strengthens the need for psychosocial support, as "CBT for adult ADHD aims to set up early success experiences that help improve engagement in treatment and hopefulness" ${ }^{21}$ (p.67). Further research into this area is needed to understand the reasons for disengaging in treatment and what the service could do or offer to help people to remain engaged in treatment.

Disparity in the levels of disengagement between those that were recently given an ADHD diagnosis (25.6\%), compared to those diagnosed prior to the present episode of care $(15.7 \%)$ highlights a potential avenue for exploring reasons for disengagement. Due to the relatively small sample size used in this evaluation, caution is needed with drawing conclusions from this finding. However, the psychological impact of both the consequences of untreated ADHD across the life span, as well as the emotional impact of receiving a diagnosis later in life, have been well documented. Matheson et al. ${ }^{22}$ highlighted that adult patients with a delayed diagnosis of ADHD have many unmet needs regarding treatment. Young ${ }^{23}$ found the delay in diagnosing ADHD until adulthood may contribute to a sense of feeling misunderstood by others, uncertainty, and dissatisfaction because the underlying cause of their problems went unidentified, misdiagnosed, and untreated for so long. He suggested a six-stage model of psychological acceptance of a diagnosis of ADHD: (a) relief and elation, (b) confusion and emotional turmoil, (c) anger, (d) sadness and grief, (e) anxiety, and (f) accommodation and acceptance. This indicates an important role for psychological treatment, which should begin at the point of diagnosis ${ }^{23}$. Comparison with a matched site that can provide this level of 
support could offer valuable insight as to the short- and long-term benefits and cost-effectiveness of offering this.

The present findings, along with the evidence discussed, highlights the wide gap between NICE guideline policy in England and clinical practice, in terms of the support that adults with ADHD receive. More investment is needed to help people to not only deal with the symptoms of ADHD they face, but to adjust to and accept a diagnosis. Having an adult diagnostic service inherently brings about a responsibility to offer an appropriate, comprehensive response to the needs of the population they serve.

\subsection{Limitations}

We are aware of the limited scope of this evaluation, which focused on the immediate impact of medication on a small number of service users' self-reported functional impairment rating. A follow-up evaluation could involve administering the WFIRS ${ }^{16}$ at annual review to see if this improvement in functional impairment continues beyond the initial episode of care.

It is important to note that interactions with clinicians in the team could constitute as an informal, nonpharmacological intervention. Caution should therefore be taken when interpreting the above results as being the result of medication alone.

The study reports on statistical significance, which could differ to a clinically significant improvement in impairment. For example, a service user reporting severe impairment at baseline may have a $50 \%$ change in symptoms, but still be symptomatic ${ }^{24}$. Weiss ${ }^{24}$ suggests that there is therefore value in looking at outcomes both from the point of view of percent change and also from the point of view of treatment endpoint.

\section{References}

1. Edwin F. A pilot service for adults with attention deficit hyperactivity disorder: A descriptive study. Adv Ment Heal Intellect Disabil . 2011;5(4):47-52. doi:10.1108/20441281111165607

2. Faraone S V., Biederman J, Mick E. The age-dependent decline of attention deficit hyperactivity disorder: A meta-analysis of follow-up studies. Psychol Med . 2006;36(2):159-165. doi:10.1017/S003329170500471X

3. Daley D, Birchwood J. ADHD and academic performance: Why does ADHD impact on academic performance and what can be done to support ADHD children in the classroom? Child Care Health Dev . 2010;36(4):455-464. doi:10.1111/j.1365-2214.2009.01046.x

4. Gjervan B, Torgersen T, Nordahl HM, Rasmussen K. Functional Impairment and Occupational Outcome in Adults With ADHD. J Atten Disord . 2012;16(7):544-552. doi:10.1177/1087054711413074

5. Nigg J. Attention-deficit/hyperactivity disorder and adverse health outcomes. Clin Psychol Rev . 2013;33(2):215-228. doi:10.1038/jid.2014.371

6. Sobanski E, Brüggemann D, Alm B, et al. Psychiatric comorbidity and functional impairment in a clinically referred sample of adults with attention-deficit/hyperactivity disorder (ADHD). Eur Arch Psychiatry Clin Neurosci . 2007;257(7):371-377. doi:10.1007/s00406-007-0712-8

7. Eakin L, Minde K, Hechtman L, et al. The marital and family functioning of adults with ADHD and their spouses. J Atten Disord . 2004;8(1):1-10. doi:10.1177/108705470400800101

8. Rösler M, Retz W, Retz-Junginger P, et al. Prevalence of attention deficit-/hyperactivity disorder (ADHD) and comorbid disorders in young male prison inmates. Eur Arch Psychiatry Clin Neurosci . 2004;254(6):365371. doi:10.1007/s00406-004-0516-Z

9. Adler LA, Spencer TJ, Levine LR, et al. Functional outcomes in the treatment of adults with ADHD. J Atten Disord . 2008;11(6):720-727. doi:10.1177/1087054707308490

10. Wender P. Attention-deficit hyperactivity disorder in adults.Psychiatr Clin North Am . 1998;21(4):761774. doi:10.7326/acpjc-2016-165-2-010 
11. National Institute for Health and Care Excellence. Attention deficit hyperactivity activity disorder: diagnosis and management. Published online 2018. https://www.nice.org.uk/guidance/cg72

12. Verity R, Coates J. Service innovation: transitional attention-deficit hyperactivity disorder clinic. Psychiatr Bull . 2007;31:99-100. doi:10.1192/pb.bp.105.0

13. Edwin F, McDonald J. Services for adults with attention-deficit hyperactivity disorder: national survey. Psychiatr Bull . 2007;31:286-288. doi:10.1192/pb.bp.10

14. Edwin MF. Adult ADHD outcome audit based on NICE guidelines.Adv Ment Heal Intellect Disabil . 2014;8(5):331-337. doi:10.1108/AMHID-08-2013-0054

15. Magon RK, Latheesh B, Müller U. Specialist adult ADHD clinics in East Anglia: service evaluation and audit of NICE guideline compliance. BJPsych Bull . 2015;39(3):136-140. doi:10.1192/pb.bp.113.043257

16. Weiss MD. Weiss Functional Impairment Rating Scale (WFIRS) instructions. Published online 2000.

17. Ramsay JR. Assessment and monitoring of treatment response in adult ADHD patients: Current perspectives. Neuropsychiatr Dis Treat . 2017;13:221-232. doi:10.2147/NDT.S104706

18. Micoulaud-Franchi JA, Weibel S, Weiss M, et al. Validation of the French Version of the Weiss Functional Impairment Rating Scale-Self-Report in a Large Cohort of Adult Patients With ADHD.J Atten Disord . 2019;23(10):1148-1159. doi:10.1177/1087054718797434

19. Canu WH, Hartung CM, Stevens AE, Lefler EK. Psychometric Properties of the Weiss Functional Impairment Rating Scale. J Atten Disord . Published online 2016:1-13. doi:10.1177/1087054716661421

20. De Crescenzo F, Cortese S, Adamo N, Janiri L. Pharmacological and non-pharmacological treatment of adults with ADHD: A meta-review.Evid Based Ment Health . 2017;20(1):4-11. doi:10.1136/eb-2016-102415

21. Ramsay JR. The relevance of cognitive distortions in the psychosocial treatment of adult ADHD. Prof Psychol Res Pract . 2017;48(1):62-69. doi:10.1037/pro0000101

22. Matheson L, Asherson P, Wong ICK, et al. Adult ADHD patient experiences of impairment, service provision and clinical management in England: A qualitative study. BMC Health Serv Res . 2013;13(1):1-13. doi:10.1186/1472-6963-13-184

23. Young S, Gray K, Bramham J. The Experience of Receiving a Diagnosis and Treatment of ADHD in Adulthood: A Qualitative Study of Clinically Referred Patients Using Interpretative Phenomenological Analysis.J Atten Disord . 2008;11(4):493-503. doi:10.1177/1087054707311659

24. Weiss MD, McBride NM, Craig S, Jensen P. Conceptual review of measuring functional impairment: Findings from the Weiss Functional Impairment Rating Scale. Evid Based Ment Health . 2018;21(4):155-164. doi:10.1136/ebmental-2018-300025

\section{Acknowledgements}

To the Norfolk and Waveney Adult ADHD Team, for their tireless commitment to improving the lives of the people they serve, and their contribution to this evaluation.

\section{Funding}

The authors received no specific funding for this work.

\section{Data availability statement}

The data that support the findings of this study are available on request from the corresponding author. The data are not publicly available due to privacy restrictions.

\section{Conflict of interest statement}

The author reports no disclosures or conflicts of interest relevant to the topic of this manuscript. 


\section{Hosted file}

ADHD table 1.pdf available at https://authorea.com/users/378927/articles/495323-a-serviceevaluation-to-determine-the-efficacy-of-a-specialist-service-for-adults-with-attentiondeficit-hyperactivity-disorder-in-norfolk-and-waveney

\section{Hosted file}

ADHD table 2.pdf available at https://authorea.com/users/378927/articles/495323-a-serviceevaluation-to-determine-the-efficacy-of-a-specialist-service-for-adults-with-attentiondeficit-hyperactivity-disorder-in-norfolk-and-waveney

\section{Hosted file}

ADHD table 3.pdf available at https://authorea.com/users/378927/articles/495323-a-serviceevaluation-to-determine-the-efficacy-of-a-specialist-service-for-adults-with-attentiondeficit-hyperactivity-disorder-in-norfolk-and-waveney 